\title{
Malignant peritoneal mesothelioma-A diagnostic and therapeutic challenge: A case report
}

\author{
Harjinder Singh ${ }^{1}$, Leyla Maric ${ }^{1}$, Neil Sharma ${ }^{2}$, Pablo A Bejarano ${ }^{3}$ and Andrew Ukleja ${ }^{4 *}$ \\ ${ }^{1}$ Department of Gastroenterology, Cleveland Clinic Florida, Weston, Florida, USA \\ ${ }^{2}$ Department of Pathology, Government medical college, Kota, Rajasthan, India \\ ${ }^{3}$ Department of Pathology, Cleveland Clinic Florida, Weston, Florida, USA \\ ${ }^{4}$ Department of Gastroenterology, Beth Israel Deaconess Medical Center, Boston, MA, USA
}

\begin{abstract}
Malignant peritoneal mesothelioma is very rare cancer of peritoneum with poor prognosis and nonspecific clinical course. We present a case of malignant peritoneal mesothelioma in a 54-year-old female who presented with chronic nonspecific upper abdominal discomfort and no occupational asbestos exposure. Abdominal CT showed mild nodularity and pelvic ascites. Paracentesis was negative for malignant cells. Diagnostic laparoscopy was performed and revealed peritoneal implants. Biopsies specimen was consistent with malignant mesothelioma-epithelioid type. Patient underwent debulking surgery and intraperitoneal chemotherapy followed by 6 months of cisplatin therapy. She is disease-free at 3 years.
\end{abstract}

\section{Introduction}

Malignant mesothelioma-neoplasia of the serosal layer arise at different sites: pleura, peritoneum, pericardium or tunica vaginalis of the testes. The most common is pleural variant. Primary peritoneal mesothelioma is rare and accounts for only $7-10 \%$ of all types of mesothelioma with an annual incidence of 800 cases/year in the United States [1]. Its association with asbestos is either occupational or nonoccupational [1-4].

\section{Case report}

A 54-year-old Hispanic female with past medical history of acid reflux and incidental painless hematuria presented in 2014 for evaluation of chronic epigastric pain, heartburn, and an episodic right lower quadrant abdominal pain. Her all other review of systems was unremarkable. She had no significant social, occupational, or gynecological history. Her complete blood count was with in normal limits. CT scan of abdomen and pelvis was done for evaluation of hematuria revealed perihepatic and pelvic fluid, epiphrenic adenopathy (largest focus was on the right and measured $2.5 \times 1.3 \mathrm{~cm}$ with smaller nodular foci at anterior inferior margin of the heart) suggestive of intra-abdominal malignancy (Figures 1 and 2). Upper endoscopy was performed in contest of the abnormal CT and was unremarkable. Cystoscopy was performed and had no significant findings. Recent colonoscopy was normal.

Diagnostic paracentesis was performed and showed chronic inflammatory changes and reactive mesothelial cells, but it was negative for malignancy. General surgery was consulted for diagnostic laparoscopy because of abnormal imaging. Diagnostic laparoscopy was performed and revealed numerous implants on serosal surface of organs, peritoneum and omentum. Histological examination of specimen collected during laparoscopy revealed epithelial malignant cells forming cords. Morphology of peritoneal mesothelioma was confirmed by performing hematoxylin and eosin stain (Figure 3) and immunohistochemical test (not shown). Immunohistochemical stains were positive for D240 (Anti-D240 antibody), calretinin, but negative for carcinoembryonic antigen (CEA) and MOC31 (monoclonal mouse antibody also known as Epithelial specific antigen) confirming the diagnosis of malignant peritoneal mesothelioma - epithelioid type.

A follow up CT scan (abdomen and pelvis) was performed later for staging of the disease, and revealed increased peritoneal nodularity along the gastrocolic ligament, and unchanged nodularity along the descending colon (Figure 4). Chest CT scan showed metastatic adenopathy. Based on the above results, cytoreduction and hyperthermic intraperitoneal chemotherapy (HIPEC) were planned. Therefore, the patient underwent debulking surgery to reduce local tumor burden, which included resection and reconstructions of right hemi-diaphragm, cholecystectomy, partial gastrectomy, distal pancreatectomy, splenectomy, right and transverse colectomy, and temporary loop ileostomy followed by intraperitoneal chemotherapy and 6-month course of cisplatin. She has been monitored closely with clinical exams and CT scans. No recurrent disease has been found at 38 months. Patient is doing well clinically.

\section{Discussion and conclusion}

Malignant peritoneal mesothelioma- creates a challenge for the medical profession in terms of rare silent disease, diagnosis, complex treatment and poor prognosis. Clinical presentation is often nonspecific which makes the diagnosis very difficult. In majority of the cases the

Correspondence to: Andrew Ukleja, Division of Gastroenterology, Beth Israel Deaconess Medical Center, 330 Brookline Ave, Boston, MA 02215, USA, Tel: 617-667-3000; E-mail: aukleja@bidco.org

Key words: Ascites, computed tomography, tumor

Received: February 16, 2018; Accepted: February 22, 2018; Published: February 28,2018 
most common symptoms and signs were bloating, abdominal pain, early satiety, weight loss, ascites, increased abdominal girth, abdominal mass, intestinal obstruction, and less commonly present: dyspnea, chest pain, dysphagia, or pleural effusion [5-7]. Diagnosis of malignant

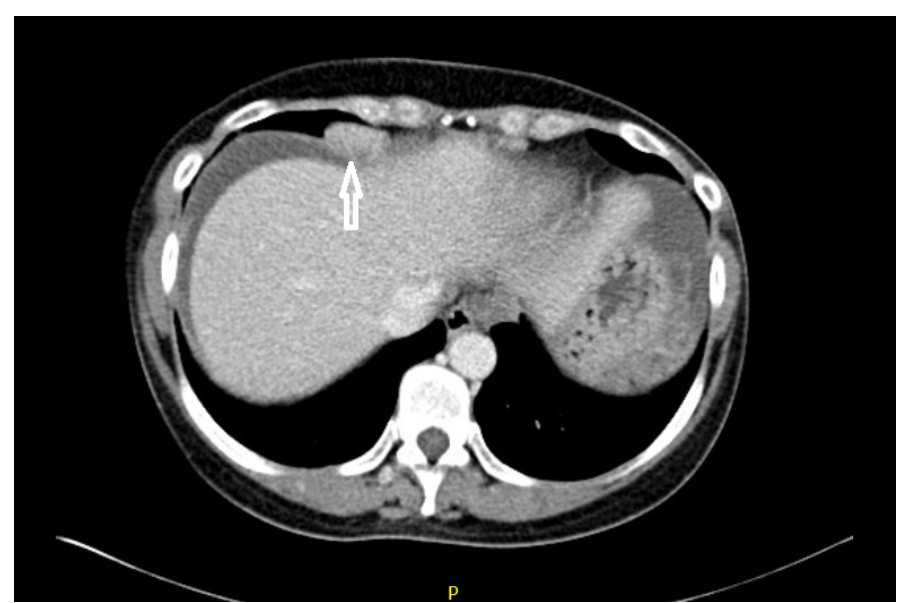

Figure 1. Showing epiphrenic adenopathy, largest focus was on the right and measured $2.5 \times 1.3 \mathrm{~cm}$ with smaller nodular foci at anterior inferior margin of the heart (shown with white arrow)

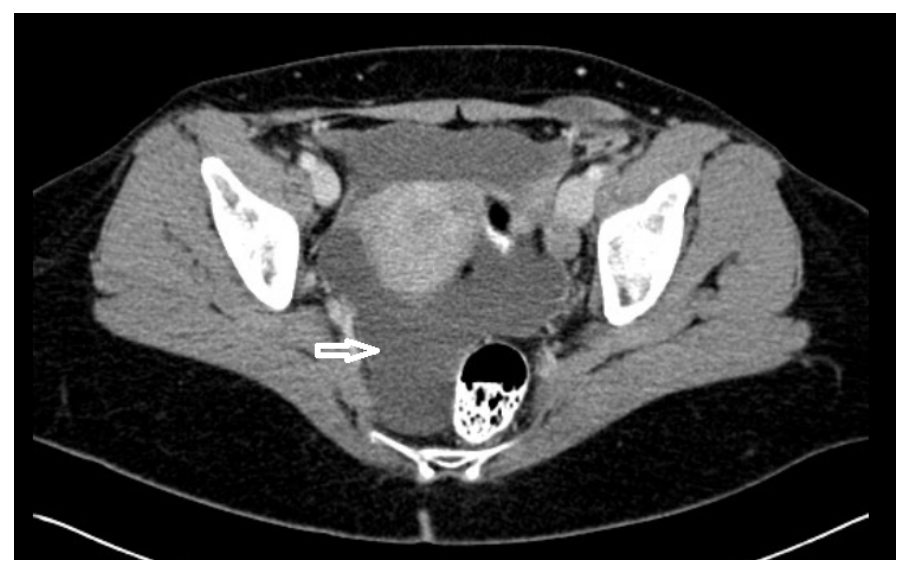

Figure 2. CT scan of abdomen and pelvis showing perihepatic and pelvic ascites (marked with white arrow).

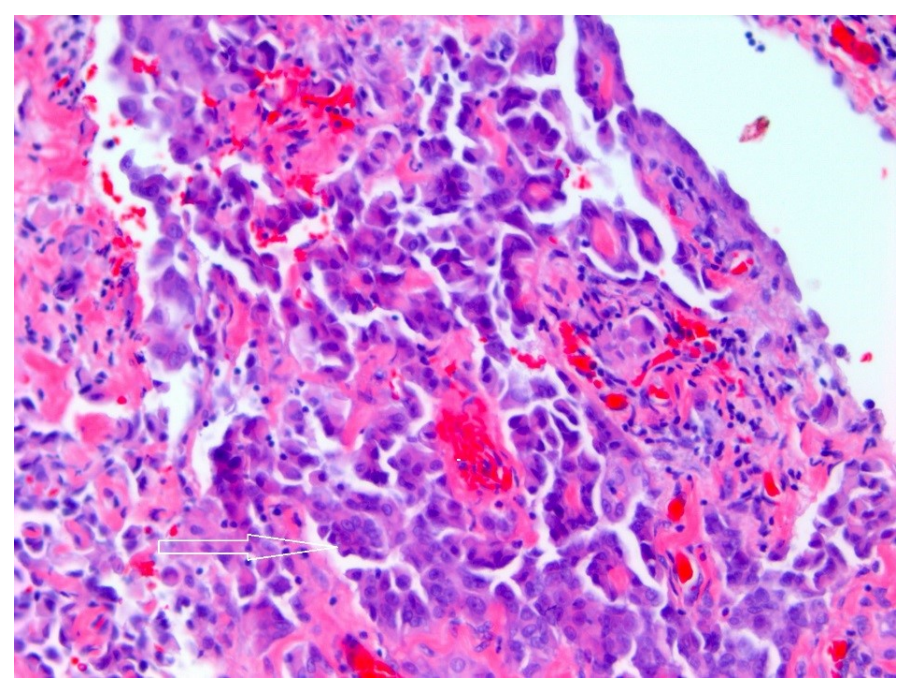

Figure 3. Histopathology of the specimen (hematoxylin and eosin stained) showing malignant cells forming cords (marked with white arrow).

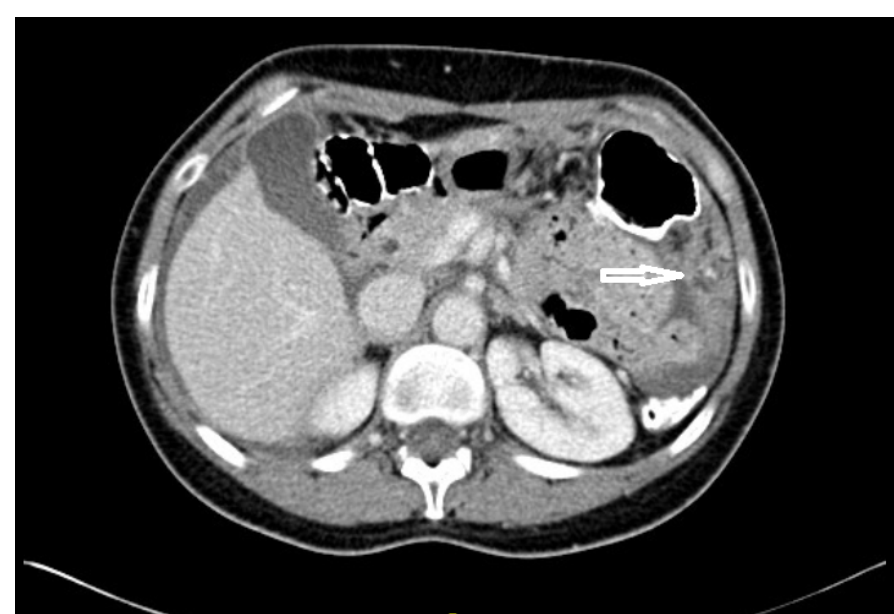

Figure 4. CT scan of abdomen showing increased peritoneal nodularity along the gastrocolic ligament (marked with white arrow), and unchanged nodularity along the descending colon.

peritoneal mesothelioma is based on high clinical suspicion of variable presentation, imaging, laparoscopy or ultrasound guided biopsy, and immunohistochemical test $[1,5,8]$. On the basis of histopathology, the mesothelioma can be divided into 3 subtypes: epithelioid (most common), sarcomatoid and tubulo-papillary [1]. CT scan findings can be broadly divided in to dry appearance; peritoneal mass and wet appearance; irregular or nodular peritoneal thickening and omental masses. Scalloping or direct invasion of adjacent abdominal organs may be seen [8]. Frozen sections of lesions (malignant peritoneal mesothelioma) may be misdiagnosed as adenocarcinoma therefore immunohistochemical diagnosis is usually needed for confirmation [5].

Survival in malignant peritoneal mesothelioma ranges from 7 to 13.5 months [7]. Elabbakh et al. [5] reported median survival of 12.5 months, while patients without chemotherapy had survival of 1 month only and those patients who undergone systemic chemotherapy had survival of 29 months [5]. Patients who underwent biopsy only had survival of 6 months as compared to patients who had debulking surgery with survival of 13.5 months. Several studies reported that combination of therapy with cytoreduction plus intraoperative chemotherapy had promising effect on patient's survival in malignant peritoneal mesothelioma. Yantaristan et al. [2] in multi institutional large registry study showed that cytoreduction followed by intraperitoneal chemotherapy had a significant impact on the survival with median survival of 53 months. One year, 3 and 5 -year survival rates were $81 \%, 60 \%$, and $47 \%$ respectively. In another large study, Feldman et al. [9] reported a median survival of 92 months with 1 year, 3 and 5-year survival rates of $86 \%, 59 \%$ and $59 \%$ respectively. Surgarbakeer et al. [10] reported 5 years survival of $44 \%$ after cytoreduction (CRS) + hyperthermic intraperitoneal chemotherapy (HIPEC), $52 \%$ post CRS+HIPEC+EPIC (early post-operative intraperitoneal chemotherapy) and $75 \%$ after CRS+HIPEC+EPIC+NIPEC (normothermic intraperitoneal and intravenous chemotherapy) which endorsed the use of long term local and systemic chemotherapy along with intraoperative chemotherapy.

Survival rate in malignant peritoneal mesothelioma is affected by multiple factors: patient's characteristics, disease stage and type of treatment. Survival is favored for female gender, young age, epithelial type, absence of lymph node metastasis or extra abdominal metastasis, CC-0(complete cytoreduction-0) or CC-1, HIPEC treatment, lack of deep invasion, lower peritoneal carcinoma index, no history of previous debulking surgery and short exposure to asbestos $[2,7,9]$. our patient's 
survival is consistent with those reported in the available literature except for the presence of lymph nodes. In our case lymph node metastases did not affect the prognosis, which is contrary to the median survival of 20 months with positive lymph nodes versus 56 months without positive lymph nodes. ${ }^{2}$ Diagnosis of malignant peritoneal mesothelioma requires high level of clinical suspicion. Therapeutic approach has to be tailored based on patient and cancer characteristics.

\section{Disclosures}

Andrew Ukleja, M.D. is a consultant for Shire.

\section{Contributions}

Harjinder Singh and Leyla Maric collected, reviewed the data and wrote the manuscript. Andrew Ukleja, Neil Sharma and Pablo A. Bejarano revised the manuscript. Harjinder Singh is the first author. Andrew Ukleja is an article guarantor. Informed consent was obtained from patient.

\section{References}

1. Alexander HR Jr, Burke AP1 (2016) Diagnosis and management of patients with malignant peritoneal mesothelioma. J Gastrointest Oncol 7: 79-86. [Crossref]

2. Yan TD, Deraco M, Baratti D, Kusamura S, Elias D, et al. (2009) Cytoreductive surgery and hyperthermic intraperitoneal chemotherapy for malignant peritoneal mesothelioma: Multi-institutional experience. J Clin Oncol 27: 6237-6242. [Crossref]
3. Faig J, Howard S, Levine EA, Casselman G, Hesdorffer M, et al. (2015) Changing pattern in malignant mesothelioma survival. Transl Oncol 8: 35-39. [Crossref]

4. Raza A, Huang W, Takabe K (2014) Advances in the management of peritoneal mesothelioma. World J Gastroenterol 20: 11700-11712. [Crossref]

5. Eltabbakh GH, Piver MS, Hempling RE, Recio FO, Intengen ME (1999) Clinical Picture, Response to Therapy, and survival of women with diffuse malignant peritoneal mesothelioma. J Surg Oncol 70: 6-12. [Crossref]

6. van Gelder T, Hoogsteden HC, Versnel MA, de Beer PH, Vandenbroucke JP, et al. (1989) Malignant peritoneal mesothelioma: a series of 19 cases. Digestion 43: 222227. [Crossref]

7. Kaya H, Sezgi C, Tanrikulu AC, Taylan M, Abakay O, et al. (2014) Prognostic factors influencing survival in 35 patients with malignant peritoneal mesothelioma. Neoplasma 61: 433-438. [Crossref]

8. Park JY, Kim KW, Kwon HJ, Park MS, Kwon GY, et al. (2008) Peritoneal mesotheliomas: Clinicopathologic features, CT findings, and differential diagnosis. AJR Am J Roentgenol 191: 814-825. [Crossref]

9. Feldman AL, Libutti SK, Pingpank JF, Bartlett DL, Beresnev TH, et al. (2003) Analysis of factors associated with outcome in patients with malignant peritoneal mesothelioma undergoing surgical debulking and intraperitoneal chemotherapy. J Clin Oncol 21 4560-4567. [Crossref]

10. Sugarbaker PH, Chang D (2017) Long-term regional chemotherapy for patients with epithelial malignant peritoneal mesothelioma results in improved survival. Eur J Surg Oncol 43: 1228-1235. [Crossref]

Copyright: (C) 2018 Singh H. This is an open-access article distributed under the terms of the Creative Commons Attribution License, which permits unrestricted use, distribution, and reproduction in any medium, provided the original author and source are credited. 